\title{
BMJ Open \\ 'Just like a normal pain', what do people with diabetes mellitus experience when having a myocardial infarction: a qualitative study recruited from UK hospitals
}

\author{
Nikita Berman, ${ }^{1,2}$ Melvyn Mark Jones, ${ }^{1}$ Daan A De Coster ${ }^{3}$
}

To cite: Berman N, Jones MM, De Coster DA. 'Just like a normal pain', what do people with diabetes mellitus experience when having a myocardial infarction: a qualitative study recruited from UK hospitals. BMJ Open 2017;7:e015736. doi:10.1136/ bmjopen-2016-015736

- Prepublication history for this paper is available online. To view these files, please visit the journal online (http://dx.doi org/10.1136/bmjopen-2016015736).

Received 6 January 2017 Revised 14 June 2017 Accepted 7 July 2017

\section{CrossMark}

${ }^{1}$ Research Department of Primary Care and Population Health, UCL Medical School, London, UK

${ }^{2}$ Dept of Psychiatry,Swn-yGwynt Day Hospital, Plymouth Hospitals NHS Trust, Plymouth, UK

${ }^{3}$ Core Medical Trainee, Princess Royal University Hospital Bromley, King's College NHS Trust, Bromley, UK

Correspondence to Dr Melvyn Mark Jones; melvyn.jones@ucl.ac.uk

\section{ABSTRACT}

Objective The objective of the study was to investigate the symptoms people with diabetes experience when having a myocardial infarction (MI), their illness narrative and how they present their symptoms to the health service.

Setting Three London (UK) hospitals (coronary care units and medical wards).

Participants Patients were recruited with diabetes mellitus (DM) (types 1 and 2) with a clinical presentation of MI (ST elevated MI (STEMI), non-ST elevated MI (NSTEMI), acute MI unspecified and cardiac arrest). A total of 43 participants were recruited, and 39 interviews met the study criteria and were analysed. They were predominantly male $(n=30)$, aged $40-90$ years and white British $(18 / 39)$, and just over a half were from other ethnic groups. The majority had type 2 DM $(n=35), 24$ had an NSTEMI, 10 had an STEMI and five had other cardiac events.

Definitions of selection/exclusion criteria A diagnosis of MI and DM and the ability to communicate enough English to complete the interview. Ward staff made a clinical judgement that the participant was post-treatment, clinically stable and well enough to participate.

Methods A qualitative study using taped and transcribed interviews analysed using a thematic analysis.

Results While most participants did experience chest pain, it was often not their most striking symptom. As their chest pain did not match their expectations of what a 'heart attack' should be, participants developed narratives to explain these symptoms, including the symptoms being effects of their DM ('hypos'), side effects of medication (oral hypoglycaemics) or symptoms (such as breathlessness and indigestion) related to other comorbidities, often leading to delays in seeking care. Conclusions While truly absent chest pain during MI among people with DM was rare in this study, patients' attenuated symptoms often led to delay in seeking attention, and this may result in delays in receiving treatment.

\section{BACKGROUND}

Diabetes mellitus (DM) is a common health problem worldwide and affects $4 \%-6 \%$ of the
Strengths and limitations of this study

- Contemporaneous patients' descriptions of their symptoms and illness narrative just after an myocardial infarction (MI).

- A model of how attenuated MI symptoms might alter help-seeking behaviour.

- The participants were from a wide demographic background in terms of age, ethnicity and disease burden.

- By recruiting patients from coronary care units, we will not have captured markedly discrepant presentations.

- By recruiting on the basis of the ability to communicate in English, we will have lost some cultural and linguistic nuance in symptom presentation.

UK population. ${ }^{1}$ Cardiovascular disease is the major life-threatening complication of DM in the UK. ${ }^{2-4}$ Myocardial infarction (MI) is the prime cause of excess mortality among those with DM, with a threefold increased risk of coronary heart disease (CHD) mortality, ${ }^{56}$ a sixfold increased risk of $\mathrm{MI}^{7}$ and a worse prognosis from MI compared with populations without diabetes. $^{8}$

Possible mechanisms of this excess mortality risk include comorbidities such as hypertension, dyslipidaemia and chronic kidney disease. An important element in this observed increased mortality may be late or missed presentation by patients and diagnosis by clinicians. ${ }^{9-11}$

Up to a third of people who suffer an MI can have no chest pain; however, people with DM make up a larger proportion of this group $\left(32.6 \%\right.$ vs $25.4 \%$ in one study) ${ }^{12}$ However, when people with DM do have symptoms of an MI, their symptoms may often be atypical ${ }^{13}$ or unusual. ${ }^{14}$ Nevertheless, there is conflicting 
evidence around this issue: Funk found no statistically significant difference in CHD symptom presentation in people with and without DM but did report non-significant increases in dyspnoea, neck and throat pain among those with DM. ${ }^{15}$ Kentsch found no differences between these groups in the frequency or severity of chest pain but also identified differences in the prevalence of dyspnoea. ${ }^{16}$ One review found conflicting results about differing MI symptoms among women with and without DM. ${ }^{17}$

There are several methodological reasons why these studies may have conflicting results: being underpowered, ${ }^{18} 19$ being unrepresentative of the population at risk $^{18}$ or being recruited from highly selected hospital populations. ${ }^{20}$ Survivor bias (ie, living to tell the tale of your symptoms) may also be an important issue in a condition such as MI which has a high early mortality. ${ }^{21}$

There is a biological basis for altered perception of pain among people with diabetes in that cardiac autonomic neuropathy is a complication of DM and leads to altered pain perception, meaning a patient might not experience pain caused by myocardial ischaemia. ${ }^{20}$

Several qualitative studies have explored patients' symptoms during MI. ${ }^{182-26}$ Within the general population experiencing MI, there are several issues identified including the considerable psychological impact of pain and specifically cardiac pain, ${ }^{27}$ cardiac symptoms that can be interpreted as pain but also can trigger symptoms such as anxiety (both as a trigger and barrier to action) and fear, ${ }^{28}$ producing responses like uncertainty ${ }^{29}$ and denial. ${ }^{30}$ Further factors identified include patients not recognising their symptoms as $\mathrm{MI}$, experiencing vague symptoms, ${ }^{31}$ misattributing their symptoms, ${ }^{23} 24$ erroneous expectations of an $\mathrm{MI}^{31}$ and the decisions patients make during their MI. ${ }^{32}$ Other factors include delays in seeking care. ${ }^{11} 30$ Theoretical models of delay in seeking treatment specifically in relation to MI have been summarised by Dracup. ${ }^{33}$ Underestimating personal risk of an $\mathrm{MI}^{23}$ and perceived lack of vulnerability to heart disease link to the concept of 'lay epidemiology' and of 'coronary candidacy', that is, not fitting individual's assumptions about the sort of person who gets a heart attack. ${ }^{34}$

There is a role of gender with MI symptoms and its interpretation; work exploring women's experience of MI symptoms found that women with diabetes had more atypical and painless MIs than men. In studies of denial among men with chest pain, there has been exploration of the impact of their gender roles adversely effecting health-seeking behaviour. ${ }^{35}$

In studies specifically exploring the experience of patient with diabetes and MI, many similar themes emerge. However, Angerud finds a variability in the experience of MI symptoms while exploring the patients' response to symptoms, perceived susceptibility and symptom interpretation (but found that MI symptoms were rarely ascribed to DM). ${ }^{25}$ Other studies in this population also identify breathlessness as a presenting symptom, misinterpretation of symptoms and their diabetes influencing patients' decision making during events. ${ }^{36}$

\section{Clinician factors}

The way clinicians ask about chest symptoms may be problematic. Clinicians ask about 'chest pain'; however, the term 'pain ${ }^{, 37} 38$ is often not used by patients experiencing MI.

In the current study, we aimed to address some of the limitations of previous research by recruiting only patients with DM with clear evidence of a recent MI, regardless of their chest pain presentation and use of a qualitative methodology to explore how these patients describe their symptoms. Qualitative methods can help overcome a narrow clinician focus and help develop a patient-oriented view when exploring symptoms of disease. ${ }^{23} 363940$ The aim of the study was to investigate the symptoms people with diabetes experience when having an MI, their illness narrative and how they present their symptoms to the health service.

\section{METHODS}

This was a qualitative study where interviews were undertaken with patients with DM who have recently had a confirmed MI, recruited within coronary care units (CCUs). Eligibility criteria included a confirmed diagnosis of MI (according to American Heart Association criteria $^{41}$ ), a clinical diagnosis of DM and the ability to communicate (understand and speak) enough English to complete the interview. Clinical staff identified participants and ensured that they were post-treatment, clinically stable and well enough to participate. This subjective judgement was made by the clinical staff involved in participants' care as required by the ethics committee. Participants were approached as soon as possible after the diagnosis by a researcher (NB) so we could be sure that they had had an MI (usually a retrospective or working diagnosis) and to minimise recall bias about symptoms. Once invited, they were given a patient information sheet about the study and 24 hours to decide whether to participate (see comments regarding ethics approval). The interviews took place in three London, UK hospitals. We aimed to recruit consecutive patients (but this proved unworkable) so this was a pragmatic sample. ${ }^{42}$

The interviews were semistructured and iterative building from a topic guide to include a narrative of the course, range, character and severity of the symptoms the person experienced before and during their MI. We used a checklist of acute MI (AMI) symptoms based on Funk's acute AMI symptom list. ${ }^{15}$ This is distinct to Diamond and Forrester's classification of angina typicality. ${ }^{43}$ The checklist was worked through to ensure that symptoms were absent and not just omitted. Ideas about expected symptoms of an MI and symptom attribution were also explored as people often develop complex narratives about their illnesses. ${ }^{44}$ We also explored participants' thoughts about their illness, treatments they had attempted, motives and triggers for seeking help and awareness and knowledge of the symptoms of a heart attack. 
Interviews were audio recorded, and field notes and baseline patient information were noted. Details gathered included the type of MI (ECG changes, troponin level and clinical assessment), the patients' demographics, comorbidities (type and duration of DM, other disease), risk factors, family history, medication use and information that might impact on symptom perception, from the informant and their medical records. All participants were allocated a code number to link their data together and then anonymised (apart from the consent form) so that identifiable data did not leave the ward setting.

The audio taped interviews were transcribed verbatim and manually analysed using a thematic framework approach, ${ }^{22}{ }^{45}$ using word processing and spreadsheet software. Agreement was specifically sought between reviewers about the classification of symptoms as either typical or atypical as outlined by Funk. ${ }^{15}$ Two independent researchers (NB, DADC) read the transcripts and analysed the data using the following steps: familiarisation, identifying a thematic framework, indexing, charting, mapping and interpretation. Throughout this process, transcripts were repeatedly re-read to focus on specific points and ideas, to verify the presence of the themes and subthemes that were identified and to ensure that the context of the themes had been preserved. There was then a dialogue between all the researchers which was reflexive in nature linking the findings back to the study's aims. The analysis was deductive or theoretical in approach in that we were looking for themes around diabetes and MI and those bounded by an expectation of probable reduced or absent pain symptomatology, and this shaped our topic guide and approach to the data. However, the analysis was also inductive in nature, in that we needed to derive themes from the data, in as much that if there were no pain symptoms during the MI, we needed the participants and their narratives to tell us what they did experience. The themes around reporting of symptoms are semantic (or a surface meaning) in that we are largely reporting the participants' descriptions of their experience. However, the explanatory models around what participants thought caused their symptoms and reactions to those symptom are much more latent thematic or interpretive analysis. ${ }^{22}$ Representative quotes (with consent) were used and have identifying details to link the theme to the participants' characteristics.

The lay term 'heart attack' is used extensively in the transcripts; this can have a broad popular meaning but we will assume that this is used by participants to suggest an MI.

Ethical approval for this study and the use of quotes was obtained from the Hertfordshire Research Ethics Committee (project 11/EE/0045). The committee requested that we allowed a 'cool off' period of 24 hours from approach to obtaining consent. Data protection and local hospital trust research and development policies were followed. Consent was not originally sought to disclose the full interview transcripts.
Patient involvement

The original idea for this study was based on a clinical encounter in general practice. A patient consulted MMJ, his general practitioner, having had a severe MI despite having minimal symptoms and subsequently developed severe heart failure. Before his death, this patient agreed to support research in this area and filmed a brief video of his experiences. A broader study (with same research question but a different methodology) on this field was presented and supported by the North East London Diabetes Research Network Lay Panel Meeting in 2011. Patients' narratives constitute the data underlying this study.

\section{RESULTS}

Forty-three participants were recruited from the participating hospitals; however, four interviews proved unusable due to not meeting study recruitment criteria on detailed case review and were excluded, resulting in a total of 39 participants' data available for analysis. Data on those who declined to participate were not recorded as it would have placed an additional burden on ward staff. The interviews had a mean duration of $20 \mathrm{~min} 33 \mathrm{~s}$ (with a range from $5 \mathrm{~min} 14 \mathrm{~s}$ to $48 \mathrm{~min} 46 \mathrm{~s}$ ) and were all undertaken by one interviewer: NB. The study comprised 30 men and nine women, with an age range of 40-90; 35 participants had type 2DM and four had type 1DM. All participants had confirmed acute coronary syndrome; 24 had non-ST elevated MIs, 10 had ST elevated MIs and five had other MIs (acute MI unspecified including four cardiac arrests) (see table 1). Their ethnic categories were white British $(\mathrm{n}=18)$, Asian/South Asian $(\mathrm{n}=7)$, African or Afro-Caribbean $(n=3)$ and others $(n=5)$ (eg, Turkish, Maltese and so on). A number $(n=6)$ declined to state their ethnicity or instead identified their religious affiliation (eg, 'Church of England', 'Jewish' and so on). One interview was done via an informal family translator but, family members would participate in the interview (in two interviews), clarifying the order of events or reminding the participant about elements of their illness narrative (which may have been clouded by their illness including cardiac arrest or treatment such as strong opioid analgesics). Participants were usually approached to participate the day following admission or the Monday following a weekend admission and then interviewed usually the following day after consenting to participate as per our ethics approval. Data saturation was achieved. Each participant was given a study number between 1 and 43 and quotes are linked (eg, participant 22 (P22)).

\section{Themes}

The analysis of the data led to the identification of the following themes and subthemes.

Symptoms

- Typical versus atypical presentations - Atypical presentations

- Absence of pain 
Table 1 Description of participants

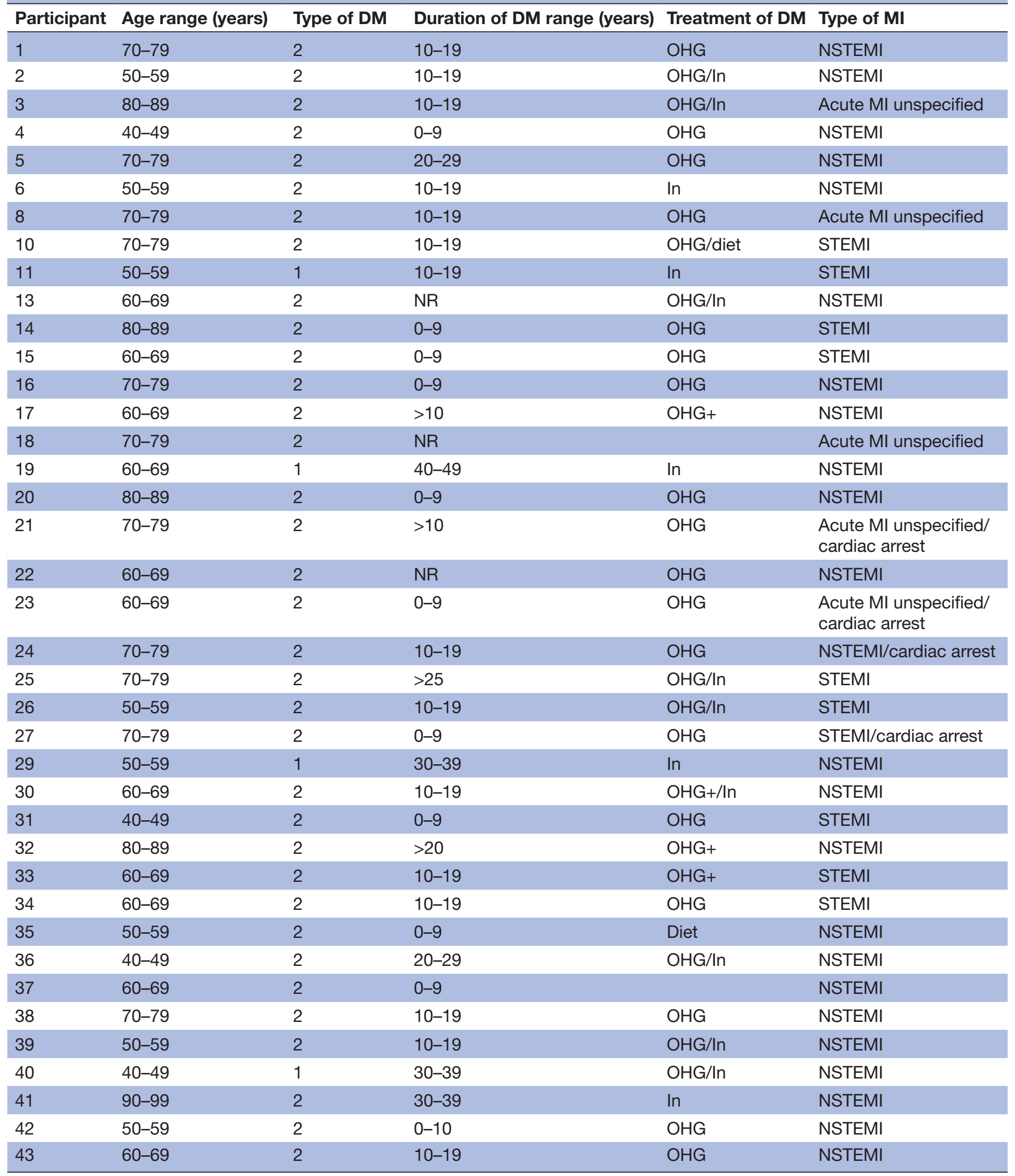

Cases 7, 9, 12 and 28 are not included as they did not meet study criteria.

Treatment key: OHG, oral hypoglycaemics (eg, metformin/glicazideand so on); In, insulins; OHG+, oral hypoglycaemics+other drugs, for example, gliptins/glitazones.

DM, diabetes mellitus; MI, myocardial infarction; NSTEMI, non-ST elevated MI; STEMI, ST elevated MI. 
Attribution and consequences

- Attributing symptoms to comorbidities

- Ascribing symptoms to DM

- Ascribing symptoms to hypoglycaemia

- Delay in seeking help

- Gradual onset of symptoms

- Multiple symptoms

- Lack of awareness of MI symptoms or their increased risk of MI

- Experience of repeat MIs

- Language of pain

- Knowing 'something was wrong'

However, these themes are not distinct. The nature of obtaining patients' narratives means that there are complex, inseparable relationships in concepts and ideas, with a continuum across themes and subthemes.

\section{Symptoms}

Participants described a wide range of symptoms, primarily around chest pain and discomfort. However, other symptoms associated with MI such as sweating and breathlessness were described, as well as a series of symptoms that are not usually associated with MI presentations. With regard to chest pain, we found a range of symptom presentations.

\section{Typical versus atypical presentations}

Chest pain of a specific character is regarded as the classic symptom of MI and was present in most of these interviews.

It was like your chest was being crushed. (P3)

Many of these descriptions catch this essence of a typical MI.

Err a real dull ache and like someone is screwing up, like a tight chest just really tight, like someone is squeezing it inside...yeah like a really, really dull pain'...'it started hurting and made me feel really ill...but then I went really pale and started sweating... absolutely saturated. just a really tight chest and sore like, as if there was a weight directly on top of me. (P 11)

Most of those in the study experienced some typical symptoms. However, typical symptoms with a recent chronology (necessary to suspect MI) were not always present or immediately apparent in the interviews.

Some experienced sweating, regarded as a typical MI symptom.

My night clothes were soaking wet. (P3).

Breathlessness was also experienced either with pain or separately, which, although classed as a 'typical' symptom [23], is not generally recognised by clinicians to be suggestive of an MI.

I can't breathe, you couldn't breathe, my wife she phone a medic... No you got the pain and you can't breathe...you think you're gonna die for the lack of breath. (P14)

Like somebody's pushing me...and I'm short of breath and then it was getting worse and it start burning. (P35)

\section{Atypical pain}

Some of those in this study sometimes narrated their experience of a diffuse and ambiguous set of symptoms that may not raise suspicion of MI.

It feels like something crawling up my arm....and it bites deep in there...the only way I can describe it, it feels like someone is in there with claws and they're tearing at your inside. Just screaming and pulling at it. Very, very strange pain indeed. (P24)

Although this person later describes quite typical sounding symptoms. Others described the quality of their pain.

It was just sticking pain, it was just a really pain, stay there for about 3 hours. (P30)

Like a normal pain, it wasn't a pain, actually it was dull. (P26)

These quotes raise the issue of participants' use of language which is discussed later.

The following quote illustrates a mixture of some typical and atypical symptoms:

I can feel something happening you know, my chest, had very bad pain in my arm, my neck and my leg, I couldn't move...Yeah sharp pain, very sharp... Somebody was pressing my, you know, chest...My back...All the way up to the waist...I couldn't breathe you know. I get very deep breath, I tried to do it but it didn't go, somebody was choking me. (P5)

The inseparability of these two types of symptoms (typical/atypical) is raised in the discussion.

Symptoms of weakness and tiredness were also reported around the timing of the MI. One participant could not walk and said,

It's just the sheer um lethargy really, I just couldn't do anything...I wasn't giddy and I wasn't in a lot of pain, I just couldn't sort of come to, I was, I couldn't put one foot in front of the other. I was just so weak. (P10)

\section{Absence of pain}

Some clearly stated they had no chest pain,

Nothing, nothing, no discomfort, no pain (pre cardiac arrest). (P27)

but this participant did have pain after being resuscitated. Another reported

No pain, no sweating, nothing else (pre cardiac arrest). (P23) 
Both of these participants experienced cardiac arrests, so some degree of event amnesia cannot be excluded.

\section{Attribution and consequences}

\section{Attributing symptoms to comorbidities}

The participants seemed to rationalise their symptoms and find a reason to dismiss that the cause of their symptoms could be a 'heart attack'. Many attributed their symptoms to other illness and causes. Most commonly, they attributed their symptoms to indigestion leading to self-medication with antacids for this,

when you feel bloated and gassy and it's similar to that sort of pain. That's why I mistook it for being indigestion.... I put it down to nerves or exercise... but then in the early hours of Sunday morning I realised it couldn't have been indigestion because it was paining me a great deal. (P31)

I took, bicarb of soda, didn't work. I took um Gaviscon (antacid remedy), didn't work. The antibiotics didn't work. That's when I knew, there was more to this. (P3)

But symptoms were also attributed to other causes that included asthma, muscular aches, panic attacks, stress and, importantly in this group, their DM.

Because of, I have other illness(es), like blood pressure, diabetes, I didn't think (of MI), because I thought there is nothing wrong with my heart. (P4).

These quotes also link to the theme of delay (both by patient and by clinician) which is discussed further on.

\section{Ascribing symptoms to DM}

Participants sometimes specifically attributed their symptoms to (lack) of control of their DM, symptoms of DM or side effects of their medication.

I didn't realise I was having heart attack, so I was feeling rotten...I was hungry. Well I wasn't really hungry but I know, don't, diabetic,...that's when the pain come, and I went to bed and it woke me up around two in the morning. (P17)

Well dizzy, tired, again I'm dizzy all the time with diabetes, you get that, that's part and parcel of being diabetic. (P17)

Participant 3 also attributed her dizziness during her MI to her DM.

Not fainting, but you know dizzy turns, because I do get these dizzy turns. You see, this is all in with the diabetes you see. (P3)

\section{Ascribing symptoms to hypoglycaemia}

Patients when experiencing some symptoms, particularly sweating and feeling unwell, perceived themselves to be experiencing hypoglycaemia ('Hypo'), for example,

because I am diabetic, right, um, I thought, initially I thought it was um low blood sugar. (P2.)
A little (of sweating) bit, but...I didn't have my testing gear with me and I thought that it might be a hypo at the time. (P11)

Participant 3 perceived herself to be experiencing hypoglycaemia.

That's when I knew, there was more to this (symptoms) and I had, I don't know whether I had five hypo's in that week, but I was rolling all over the place. (P3).

\section{Delay in seeking help}

Delay in help seeking was a prominent feature in these narratives.

I think to be honest it (chest pain) came on suddenly but I tended to ignore it, I've got one more screw to put in. (P21)

I waited till my GP opened in the morning. I called him and I told him what's wrong, they said 'just phone the ambulance. (P5).

This delay could be ascribed to participants' failure to attribute symptoms to MI, attribution to comorbidities, lack of symptom awareness or lack of awareness of increased risk of MI, but participants often realised that something more serious was occurring when their symptoms did not improve.

But it was there too long, so I said 'could be heart attack. (P30)

Often participants had dismissed or explained away these one off or minor symptoms and did not think much of them, except with hindsight.

more during the week. Nothing sharp...it was just a niggling thing, it just came and then it went away... you just didn't think too much of any of these factors in isolation; but together. (P31)

With others, denial was behind their delay.

Well I thought it was a heart attack but I didn't really want to admit it at the time. Anything other than a heart attack. (P17)

\section{Gradual onset of symptoms}

For some participants, part of this delay was the gradual rather than sudden onset of symptoms.

'Well no...it had happened a fortnight before'...'Sat in the chair for about $20 \mathrm{~min}$ and then it went so then I said to my wife, I told her 'If it happens again I would be calling the doctor'. (P20)

\section{Multiple symptoms}

The respondents often had complex narratives where chest pain was a part of the symptom complex they described, but their chest pain symptom may not have been the most prominent, important or distressing feature, exemplified by the following quote. 
It (chest pain) did come on suddenly... at the same time I had this very heavy perspiration... and this very uncomfortable tummy ache...Err all three things hitting me at once; you don't pick one out in particular...all those three factors the er tummy ache the excessive err sweating and the pain in the chest um I could point to one and say look that was the main problem. they were all a problem at that short period of time. (P 25)

\section{Lack of awareness of Ml symptoms or their increased risk of Ml} Many of these respondents, despite having long-standing diabetes, previous cardiac events or a strong family history, seemed unaware that they were at a high risk of subsequent MI.

I thought...how can I have a heart attack? Right. But you see, this might be relevant as well; both my side of my family, my mother's side and my father's side are diabetic. My father had a heart attack as well and he died from it. My mother had a triple heart bypass. My elder brother's had an angioplasty, right, so it does run in the family. (P2)

\section{Experiences of a repeat MI}

Participants who had previous MIs might be expected to have a good working knowledge of these symptoms and respond accordingly if it recurred. We found, however, that often they did not do so.

I should have guessed, right, um, it's exactly the same symptoms as when I had my first heart attack. (P2)

\section{Language of pain}

The expectation of severe chest pain is key to many patients' and doctors' model of what an MI should be like and is apparent in some of the previous themes. However, many of these respondents had symptoms that they did not feel reached a threshold of severity that they would call 'pain'.

I suppose people would call it a pain, I don't know, I wouldn't, I would still call it severe discomfort...dull, rather than, no it wasn't sharp.... I won't put it (pain severity score) higher, otherwise I probably would have called an ambulance straight away. (P20)

No, it was a constant. When I say pain, ache, it wasn't a pain that was making me double up or anything. (P25)

While some struggled to conceptualise their symptoms as pain, others struggled to use words to describe their symptoms.

It's so difficult cos it was more uncomfortable than a pain that you can describe...I don't know how to describe it. (P20)

While others clearly had very significant pain but still struggled to articulate it,
Indescribable to me, I couldn't, as I sit here I can't describe it...I had severe pain and it was; I don't know how you break it down into words. (P19)

\section{Knowing 'something is wrong'}

Frequently participants had a 'gut feeling' that there was something wrong or a strong emotional response to their symptoms.

You know, I could sort of feel that there was something wrong. (P2).

And I thought I can't do this, I got to get 'elp', and that's when I dialled $999 \ldots$ and, you know, everything was going wrong, and I just couldn't figure out. (P3).

A 'sense of doom' is often associated with the presentation of MI, but in this group, only a few participants reported a strong sense of impending death.

I felt as if I was going to die (anxious voice). (P6)

\section{DISCUSSION}

We found that patients with DM who experienced an MI exhibited a wide range of symptoms from very classical presentations to very mild symptoms. ${ }^{23}$ Patients often were unclear what caused their symptoms. ${ }^{46}$ This diagnostic confusion or uncertainty appears periodically to have been mirrored by clinicians. ${ }^{924}$

The symptoms experienced in this group ranged from those that may be expected by healthcare professionals to more atypical symptoms with patients using unusual descriptive terms. The variation in experiences highlights the difficulty for both patients and healthcare providers to correctly attribute these symptoms to a cardiac event.

Patients with DM will often have other significant comorbidities which can be attributed to their symptoms, for example, breathlessness from chronic obstructive pulmonary disease rather than an MI. However, there seems to be an important area of confusion from symptoms related to DM and its control, that can lead to a misattribution of pain symptoms away from a new onset MI. A specific issue among this group is the impact of MI symptoms which were perceived as a side effect of medication (particularly insulin and oral hypoglycaemics). Interpreting episodes of hypoglycaemia and sudden onset MI seems to be particularly challenging with an overlap of many similar symptoms and their sudden onset. ${ }^{25}$

We found that participants had a lack of knowledge of MI symptoms, were falsely reassured by the lack of severe pain or did not conceptualise their milder chest discomfort as chest pain. These factors may all lead to denial or confusion and therefore delay in seeking care. ${ }^{33}$ Delay in seeking medical attention during an MI will have significant prognostic implications.

As the participants' symptoms did not fit with their preconceived ideas of an MI, ${ }^{25}$ they ascribed it to other less serious causes, or importantly attributed their symptoms 
to their DM, medication side effects or 'hypos', which is a novel and important finding. The attenuated chest pain competing with other, perhaps more distressing symptoms and so being 'crowded out' from the illness cognition of a possible MI, has not been previously reported.

\section{Strengths}

Within this study, we have reported contemporaneous symptoms from a well-described patient group, who can be characterised by their MI diagnosis. We have captured a wide range of participants with MI, by using biochemical confirmation of diagnosis wherever possible and by including clinical diagnoses (eg, for cardiac arrests). Unusually for a qualitative study, we have a relatively large number of participants, who were interviewed as early as possible (or allowed to) after the event to reduce recall bias. $^{47}$

\section{Limitations}

Despite recruiting participants on the basis of their MI diagnosis, but not specifically on chest pain presentations, participants are likely to have initially volunteered chest pain symptoms to get admitted to a CCU. Markedly discrepant MI narratives are less likely to be represented in this study, as are silent MIs, as they are more likely to go unrecognised and present late. Survivor bias is also a distinct possibility. ${ }^{21}$ While a study size of 39 is good for qualitative research, ${ }^{47}$ it is small in comparison with epidemiological studies so this methodology is therefore unlikely to pick up rarer presentations of MI.
Recruiting on the basis of being able to converse in English will exclude the linguistic nuance of other languages and cultures, important when South Asian populations among others have a high risk of DM and CHD. However, we did successfully capture many patients who had English as second language. In addition, the UK National Health Service struggles to provide reliable, accessible translation, particularly, in the acute and out-of-hours setting, so our findings may more faithfully reflect the real-world setting of these decisions and presentations.

Truncating these interviews into quotes also runs the danger of falsely dichotomising symptoms into 'typical' and 'atypical'. When the transcripts are read in full and in context, a more rounded picture of the event develops. This nuanced view is probably a key element that helps experienced clinicians successfully identify a potential MI, in this group of patients.

\section{How this fits into existing literature}

The findings of our study link with previous work within a broad context of psychological models of illness behaviour such as health belief models. ${ }^{48}$ We have used our findings to adapt Strecher, Rosenstock and Kirsch's health belief model $^{48}$ (see figure 1) so that the impact of DM can modify the steps in the model such as reduced pain from neuropathy during an MI reducing the likelihood of action.

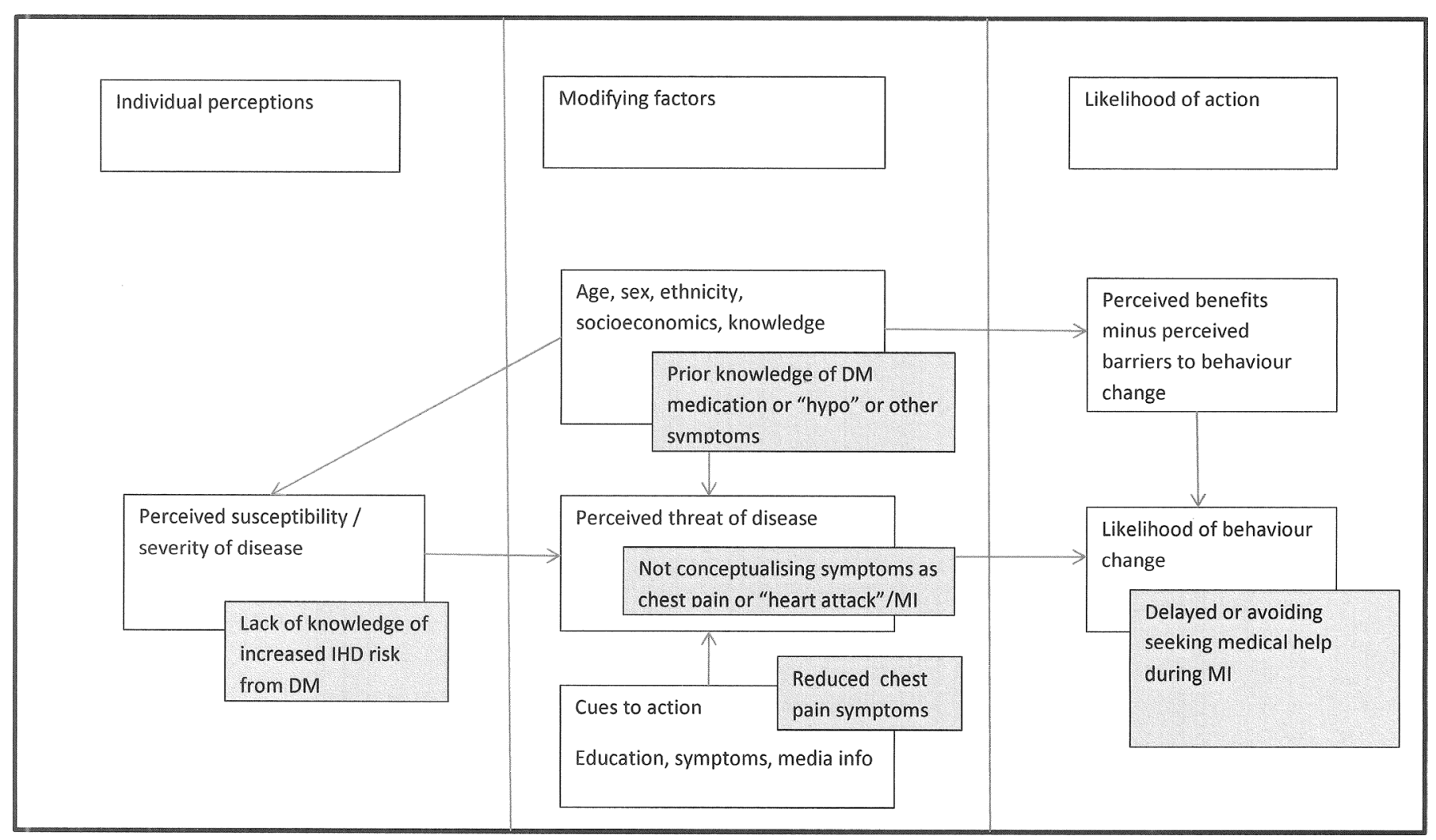

Figure 1 How diabetes (grey boxes) might affect the 'health belief model' during MI. (adapted from Strecher and Rosenstock. ${ }^{48}$. DM, diabetes mellitus; IHD, ischaemic heart disease; MI, myocardial infarction. 
The language of describing pain and particularly the difficulty of describing pain recurs within our sample. Scarry describes this as the "inexpressibility of pain ${ }^{49}$ and this is seen with some respondents. Others use metaphor and simile to circumvent this issue, which may be helpful to the patient but means that a clinician might cognitively process such symptom descriptions as 'atypical' or 'non-cardiac', leading to misdiagnosis and delays in treatment. The term 'chest pain' is also problematic ${ }^{37}$; many of these participants did not conceptualise their chest symptoms as pain and so Miller has suggested using the term 'chest sensation' instead..$^{50}$

Our findings have some similarities to other studies about patients' experiences of MI. ${ }^{23-26}$ The similarity of symptom presentation raises the possibility that these studies are just capturing the heterogeneous array of MI symptoms and this is unlinked to any underlying physiological or pathological process such as gender or DM. However, our finding of patient misattribution of MI symptoms to DM and diabetic medication, in a group at high risk of MI, does have significant clinical implications for patients and clinicians.

\section{Implications for further research}

Despite knowing that people with DM are at much higher risk of coronary events, we fail to inform patients in the UK about their increased cardiovascular risk nor identify early those who may be developing such problems. Whether screening people with diabetes for ischaemic heart disease would be beneficial remains a key question. Possibilities include trialling patient education about their CHD risk and its presentation in the UK Quality and Outcome (Quality and Outcomes Framework) DM check or including this information within DESMOND (desmond-project.org.uk)/DAFNE (www.dafne.uk.com) patient education programmes, but there would be considerable difficulty in designing an educational package that could reliably help patients distinguish between these two aetiological possibilities of 'hypo' versus MI.

\section{Implications for practice}

This study could contribute towards increased awareness of MI in those with DM. Patients with DM should be made aware of their increased risk of cardiac events, how they present and how they differ from medication side effects and 'hypos'. Clinicians should carefully explore the symptoms of patients with diabetes as well as their interpretation of their symptoms.

Contributors All three authors fulfil all three of the International Committee of Medical Journal Editors guidelines for authorship. MMJ had the original study conception and design and then supervised NB during her iBSc project. NB collected all the data. NB and DADC analysed the final dataset. MMJ wrote the final manuscript with substantial intellectual input from NB and DADC. All authors approved the final manuscript.

Funding Royal College of General Practitioners' Scientific Foundation Board (grant SFB 2011-25). The original project was part of the University College London iBSC in primary healthcare and formed NB's dissertation, supervised by MMJ.

Competing interests None declared.
Ethics approval National Research Ethics Service Committee East of England, Hertfordshire.

Provenance and peer review Not commissioned; externally peer reviewed.

Data sharing statement № additional data are available as participants only consented to quotes being published.

Open Access This is an Open Access article distributed in accordance with the Creative Commons Attribution Non Commercial (CC BY-NC 4.0) license, which permits others to distribute, remix, adapt, build upon this work non-commercially, and license their derivative works on different terms, provided the original work is properly cited and the use is non-commercial. See: http://creativecommons.org/ licenses/by-nc/4.0/

(C) Article author(s) (or their employer(s) unless otherwise stated in the text of the article) 2017. All rights reserved. No commercial use is permitted unless otherwise expressly granted.

\section{REFERENCES}

1. González EL, Johansson S, Wallander MA, et al. Trends in the prevalence and incidence of diabetes in the UK: 1996-2005. J Epidemiol Community Health 2009;63:332-6.

2. Wannamethee SG, Shaper AG, Lennon L, et al. Cardiovascular disease incidence and mortality in older men with diabetes and in men with coronary heart disease. Heart 2004;90:1398-403.

3. Shah AD, Langenberg C, Rapsomaniki E, et al. Type 2 diabetes and incidence of cardiovascular diseases: a cohort study in 1.9 million people. Lancet Diabetes Endocrinol 2015;3:105-13.

4. The Emerging Risk Factors Collaboration. Diabetes mellitus, fasting glucose, and risk of cause-specific death. New England Journal of Medicine 2011;364:829-41.

5. Huxley R, Barzi F, Woodward M, et al. Excess risk of fatal coronary heart disease associated with diabetes in men and women: metaanalysis of 37 prospective cohort studies. BMJ 2006;332:73-8.

6. Stamler J, Vaccaro O, Neaton JD, et al. Diabetes, other risk factors, and 12-yr cardiovascular mortality for men screened in the multiple risk factor intervention trial. Diabetes Care 1993;16:434-44.

7. Haffner SM, Lehto S, Rönnemaa T, et al. Mortality from coronary heart disease in subjects with type 2 diabetes and in nondiabetic subjects with and without prior myocardial infarction. N Engl J Med 1998;339:229-34.

8. Brophy S, Cooksey R, Gravenor MB, et al. Population based absolute and relative survival to 1 year of people with diabetes following a myocardial infarction: a cohort study using hospital admissions data. BMC Public Health 2010;10:338.

9. Sequist TD, Marshall R, Lampert S, et al. Missed opportunities in the primary care management of early acute ischemic heart disease. Arch Intern Med 2006;166:2237-43.

10. Asaria $P$, Elliott $P$, Douglass $M$, et al. Acute myocardial infarction hospital admissions and deaths in England: a national follow-back and follow-forward record-linkage study. Lancet Public Health 2017;2:e191-e201.

11. Ängerud $\mathrm{KH}$, Brulin $\mathrm{C}$, Näslund $\mathrm{U}$, et al. Longer pre-hospital delay in first myocardial infarction among patients with diabetes: an analysis of 4266 patients in the northern Sweden MONICA Study. BMC Cardiovasc Disord 2013;13:6.

12. Canto JG, Shlipak MG, Rogers WJ, et al. Prevalence, clinical characteristics, and mortality among patients with myocardia infarction presenting without chest pain. JAMA 2000;283:3223-9.

13. Brieger D, Eagle KA, Goodman SG, et al. Acute coronary syndromes without chest pain, an underdiagnosed and undertreated high-risk group: insights from the global registry of acute coronary events. Chest 2004;126:461-9.

14. Arslanian-Engoren $\mathrm{C}$, Patel $\mathrm{A}$, Fang J, et al. Symptoms of men and women presenting with acute coronary syndromes. Am J Cardiol 2006;98:1177-81.

15. Funk M, Naum JB, Milner KA, et al. Presentation and symptom predictors of coronary heart disease in patients with and without diabetes. Am J Emerg Med 2001;19:482-7.

16. Kentsch M, Rodemerk U, Gitt AK, et al. Angina intensity is not different in diabetic and non-diabetic patients with acute myocardial infarction. Z Kardiol 2003;92:817-24.

17. Stephen SA, Darney BG, Rosenfeld AG, et al. Symptoms of acute coronary syndrome in women with diabetes: an integrative review of the literature. Heart Lung 2008;37:179-89.

18. DeVon HA, Penckofer SM, Zerwic JJ, et al. Symptoms of unstable angina in patients with and without diabetes. Res Nurs Health 2005;28:136-43. 
19. Richman PB, Brogan GX, Nashed AN, et al. Clinical characteristics of diabetic vs nondiabetic patients who "rule-in" for acute myocardial infarction. Acad Emerg Med 1999;6:719-23.

20. Airaksinen KE, Koistinen MJ. Association between silent coronary artery disease, diabetes, and autonomic neuropathy. fact of fallacy? Diabetes Care 1992;15:288-92.

21. Saracci R. Survival-related biases survive well. Int J Epidemiol 2007;36:244-6.

22. Braun V, Clarke V. What can "thematic analysis" offer health and wellbeing researchers? Int J Qual Stud Health Well-being 2014;9:26152.

23. Lichtman JH, Leifheit-Limson EC, Watanabe E, et al. Symptom recognition and healthcare experiences of young women with acute myocardial infarction. Circ Cardiovasc Qual Outcomes 2015;8:S31-S38.

24. Coventry LL, van Schalkwyk JW, Thompson PL, et al. Myocardial infarction, patient decision delay and help-seeking behaviour: a thematic analysis. J Clin Nurs 2017;26:1993-2005.

25. Ängerud $\mathrm{KH}$, Brulin C, Eliasson M, et al. The process of Care-seeking for myocardial infarction among patients with Diabetes. J Cardiovasc Nurs 2015;30:E1-E8.

26. DeVon HA, Hogan N, Ochs AL, et al. Time to treatment for acute coronary syndromes: the cost of indecision. J Cardiovasc Nurs 2010;25:106-14.

27. MacDermott AF. Living with angina pectoris--a phenomenological study. Eur J Cardiovasc Nurs 2002;1:265-72.

28. Tod AM, Read C, Lacey A, et al. Barriers to uptake of services for coronary heart disease: qualitative study. BMJ 2001;323:214.

29. Fitzsimons D, Parahoo K, Richardson SG, et al. Patient anxiety while on a waiting list for coronary artery bypass surgery: a qualitative and quantitative analysis. Heart Lung 2003;32:23-31.

30. Johansson I, Swahn E, Strömberg A, et al. Manageability, vulnerability and interaction: a qualitative analysis of acute myocardial infarction patients' conceptions of the event. Eur $J$ Cardiovasc Nurs 2007;6:184-91.

31. Nymark C, Mattiasson AC, Henriksson $P$, et al. The turning point: from self-regulative illness behaviour to care-seeking in patients with an acute myocardial infarction. J Clin Nurs 2009;18:3358-65.

32. Pattenden J, Watt I, Lewin RJ, et al. Decision making processes in people with symptoms of acute myocardial infarction: qualitative study. BMJ 2002;324:1006.

33. Dracup K, Moser DK, Eisenberg M, et al. Causes of delay in seeking treatment for heart attack symptoms. Soc Sci Med 1995;40:379-92.

34. Emslie C, Hunt K, Watt G, et al. 'I'd rather go with a heart attack than drag on': lay images of heart disease and the problems they present for primary and secondary prevention. Coronary Health Care 2001;5:25-32.

35. White AK, Johnson M. Men making sense of their chest pain-niggles, doubts and denials. J Clin Nurs 2000;9:534-41.

36. Mayer DD, Rosenfeld A. Symptom interpretation in women with diabetes and myocardial infarction: a qualitative study. Diabetes Educ 2006;32:918-24.

37. Treasure T. Pain is not the only feature of heart attack. BMJ 1998;317:602.

38. Albarran JW, Clarke BA, Crawford J, et al. 'It was not chest pain really, I can't explain it!' An exploratory study on the nature of symptoms experienced by women during their myocardial infarction. J Clin Nurs 2007;16:1292-301.

39. Farmer SA, Higginson IJ. Chest pain: physician perceptions and decision-making in a London emergency department. Ann Emerg Med 2006;48:77-85.

40. Jones MM, Somerville C, Feder G, et al. Patients' descriptions of angina symptoms: a qualitative study of primary care patients. $\mathrm{Br} J$ Gen Pract 2010;60:735-41.

41. Thygesen K, Alpert JS, White HD, et al. Universal definition of myocardial infarction. Circulation 2007;116:2634-53.

42. Emmel N. Sampling and choosing cases in qualitative research: a realist approach. London: Sage, 2013.

43. Diamond GA, Forrester JS. Analysis of probability as an aid in the clinical diagnosis of coronary-artery disease. $N$ Engl J Med 1979;300:1350-8.

44. Kleinman A. The illness narratives: suffering, healing, and the human condition: Basic books, 1988

45. Ritchie J, Spencer E. Qualitative data analysis for applied policy research. London: Routledge, 1994.

46. Kirchberger I, Heier M, Wende R, et al. The patient's interpretation of myocardial infarction symptoms and its role in the decision process to seek treatment: the MONICA/KORA Myocardial Infarction Registry. Clin Res Cardiol 2012;101:909-16.

47. Baker SE, Edwards R. How many qualitative interviews is enough? expert voices and early career reflections on sampling and cases in qualitative research. secondary how many qualitative interviews is enough? expert voices and early career reflections on sampling and cases in qualitative research. 2012. http://eprints.ncrm.ac.uk/2273/4/ how_many_interviews.pdf

48. Strecher VJ, Rosenstock IM. The health belief model. Cambridge handbook of psychology, health and medicine, 1997:113-7.

49. Scarry E. The body in pain. New York: Oxford University Press, 1985

50. Miller CL. Symptom reflections of women with cardiac disease and advanced practice nurses: a descriptive study. Prog Cardiovasc Nurs 2003;18:69-76. 